\title{
Intraoperative Plasma: Rbc Ratio Affects Postoperative Coagulopathy And Resuscitation Requirements
}

\author{
Christopher V Maani*, Ethan Scott, Peter A Desocio, Laura L McGhee, Thomas Garza, Evan M Renz, Steven E Wolf, John B Holcomb and \\ Lorne H Blackbourne
}

Home of the Joint Center of Excellence for Battlefield Health and Trauma Research, USA

\section{Background}

Early and increased amounts of plasma have been associated with improved survival after penetrating and blunt injury. However, no studies involving burn patients demonstrate the effects of intraoperative plasma administration on postoperative resuscitation requirements. This study examined perioperative transfusion ratios [plasma:RBC $(\mathrm{P}: \mathrm{R})]$ and the role of early, aggressive plasma administration in a contemporary burn center.

\section{Methods}

We performed a single-center, 6-month retrospective analysis of 148 operative interventions beginning January 2007. Inclusion criteria were operations in burn patients $>18$ years of age with $>20$ percent TBSA burn who incurred intraoperative EBLs $>500 \mathrm{~mL}$. Data was obtained from medical records, trauma registry and the institution's blood bank. Since surgical interventions for extensive burns are often done as staged procedures, individual patients sometimes do appear in the study as multiple different entries. The data were divided into a high plasma:RBC group $(>1: 2)$ and a low plasma:RBC $(<1: 2)$ group. These groups were then evaluated using a Wilcoxon Signed Rank test for postoperative transfusion requirements for both plasma and red blood cells for the 24-hour postoperative period.

\section{Results}

The typical patient included in the study was a 29 -year-old male with 52 percent TBSA; 42 percent of which was full thickness. The average surgery lasted 133 minutes and had an EBL of $1458 \mathrm{~mL}$. Mean intraoperative transfusion included 1.24 units plasma and 2.82 units RBCs; however, mean blood product administration in the postoperative period was 0.99 units plasma and 0.95 units RBCs. An analysis of the two groups showed that both the high plasma and the low plasma groups were similar in regards to age, gender, and ASA classification. No statistical difference was apparent when comparing preoperative hematocrit, postoperative hematocrit or postoperative INR. Preoperative INR was statistically greater in the higher P:R cohort, and these patients received more albumin and platelets postoperatively $[p<0.05]$. Patients with interoperative P:R $>1: 2$ were also statistically more likely to have longer operations. Analysis of our data showed that patients who received a higher ratio of plasma required fewer transfusions of both red blood cells and plasma postoperatively and that both of these results reached statistical significance. Results also showed that in the 24 hours following surgery, the mean P:R ratio jumped from 0.44 interoperatively to 1.04 postoperatively.

\section{Discussion}

The debate over the ideal large volume resuscitation is fraught with conflicting information and subject to passionate debate. Certainly, tomes could be written about the crystalloid versus colloid discussion itself, but that review is beyond the scope of our study. The question of when to start transfusing plasma and at what ratio to packed cells has also been longstanding. In fact, the debate over proper use of fresh frozen plasma has been ongoing for more than 20 years. After a 10 -fold increase in FFP administration between the years of 1974-1984, the National Institutes of Health convened a Consensus Development Conference on Fresh Frozen Plasma [1]. The recommendations that came from the conference said that that FFP was indicated for replacement of isolated factor deficiencies, coumadin reversal, antithrombin III deficiency, treatment of thrombotic thrombocytopenic purpura and massive blood transfusion. Massive blood transfusion was defined as blood loss greater than one blood volume over the course of several hours. The consensus statement made no mention of what ratio of FFP to packed red blood cells was recommended [2].

Subsequently, the American Society of Anesthesiologists published recommendations in 2006 from its transfusion task force. The guidelines for FFP cover just two paragraphs and limit the use of FFP to five indications. The first is microvascular bleeding along with abnormal coagulation tests (PT 2 times normal, INR greater than 2.0 or aPTT greater than 2 times normal). The second indication is microvascular bleeding secondary to coagulation factor deficiency in patients who have had more than one blood volume of transfusion when results from coagulation studies cannot be made available quickly. Other indications included urgent coumadin reversal, correction of known coagulation factor deficiencies and antithrombin III deficiency in a patient needing heparin treatment. At any rate, the ASA recommendations would seem to preclude giving FFP until a patient has an abnormal coagulation study or until the patient has been transfused at least one blood volume worth of red blood cells. Once the criteria for FFP transfusion has been met, the ASA task force recommended transfusing up to a minimum of 30 percent of plasma factor concentration (hence the 1:3 FFP to PRBC transfusion ratio mentioned in some other articles) [3].

Both the NIH consensus and the ASA recommendations note the potential for infection and the fact that PT and PTT may remain normal with less than a $50 \%$ concentration of coagulation factors in support of the position that plasma transfusion should initially be avoided. The problem arises that patients suffering massive hemorrhage are often already coagulopathic on arrival at an emergency department and that massive transfusion protocols based on the previously listed recommendations do not adequately treat this coagulopathy [4].

${ }^{*}$ Corresponding author: Christopher V. Maani MD, Chief of Anesthesia, U.S Army Institute of Surgical Research, Army Burn Center \& Pain Research Area, Fort Sam Houston, TX 78234, Tel: 210-916-1044; E-mail: Christopher.Maani@ AMEDD.ARMY.MIL

Received March 28, 2011; Accepted March 28, 2011; Published March 31, 2011

Citation: Maani CV, Scott E, Desocio PA, McGhee LL, Garza T, et al. (2011) Intraoperative Plasma: Rbc Ratio Affects Postoperative Coagulopathy And Resuscitation Requirements. J Blood Disord Transfus 2:e101. doi:10.4172/21559864.1000e101

Copyright: ( 2011 Maani CV, et al. This is an open-access article distributed under the terms of the Creative Commons Attribution License, which permits unrestricted use, distribution, and reproduction in any medium, provided the original author and source are credited. 
Because of these findings, Gonzalez et al. [4] recommended changing the massive transfusion protocol to emphasize the early administration of fresh frozen plasma. The previous recommendations of withholding plasma until six units of packed cells had been transfused were scrapped in favor of a protocol calling for 1:1 plasma to red blood cell transfusion ratio. Another study showed that the rate of death by exsanguinations for patients with pelvic fractures dropped from 9 percent to 1 percent after changing the transfusion protocol to allow for administration of plasma in the emergency department [5].

Avoiding the transfusion of plasma until a full volume of red cells has been replaced is not the only recommendation that has been challenged. There are multiple papers from both military and civilian medical centers that deem the 1:3 plasma to RBC transfusion ratio inadequate. One retrospective study of patients in a combat support hospital found that a transfusion ratio of plasma to RBCs of around 1:1 led to an increased rate of survival. This survival benefit was mainly due to decreased death secondary to hemorrhage [6]. Another study involving combat casualties also showed benefits of improved hemostasis and shorter ventilator times, in addition to increased survival when using either a 1:1 ratio of plasma to RBCs or fresh whole blood [7]. Another retrospective military study looking at all patients who received blood component therapy while at one combat support hospital found that FFP transfusion amounts were independently associated with increased survival. Likewise, increasing amounts of RBC transfusions were independently associated with decreased survival [8]. Such findings might accentuate the utility of transfusing plasma early to decrease the chances of coagulopathy and need for increasing RBC transfusions.

While military research recommending early and increased use of plasma has been plentiful, there is also civilian data fueling the controversy. At least two larger retrospective studies have also concluded that plasma to RBC transfusion ratios near 1:1 confer survival benefits on trauma patients who undergo massive transfusion. One study included 466 mass transfusion patients who were treated at 16 level 1 trauma centers. Patients were divided into high $(>1: 2)$ plasma to $\mathrm{RBC}$ and low $(<1: 2)$ plasma to $\mathrm{RBC}$ groups and were then evaluated for outcome. Patients in the high plasma to RBC group were determined to have improved survival at 6 hours, 24 hours and 30 days [9]. The second study included 626 trauma patients at one trauma center who received blood transfusions over the course of four years. This study found a statistically significant improvement in mortality when patients who received a massive transfusion ( $>10$ units RBCs) also received plasma at a 1:1 ratio. This study also found a trend toward decreased mortality for patients who received $<10$ units RBCs along with plasma at a 1:1 ratio, though this finding was not statistically significant [10].

These findings have not been replicated universally in the civilian setting. A retrospective analysis of 166 patients who underwent massive transfusion at a single trauma center found that while plasma to RBC ratio of 1:1 led to statistically significant improvement in coagulopathy, there was no difference in mortality compared with patients who received a lower ratio [11]

While there is a multitude of data regarding plasma transfusion in blunt and penetrating trauma, there is little data to suggest that early and increased plasma might be of benefit to burn patients. Other facets of burn resuscitation, particularly the initial stages of resuscitation, have been frequently studied. Recommendations for an initial infusion of crystalloid at volumes of $2-4 \mathrm{ml} / \mathrm{kg} / \%$ burn for the first 24 hours have been well publicized, though they are certainly not without controversy
[12]. We do not address this topic whatsoever in our research. Many of our patients are soldiers who received their initial care in theater and then were transferred to our facility for definitive care. None of our data was gathered from initial burn resuscitation.

While burn patients may not have initial hemorrhage (though many of our military patients do have other injuries that cause immediate hemorrhage), all patients who require large scale excision and skin grafting may become candidates for blood transfusions. Considering that our average estimated blood loss for this study was nearly $1.5 \mathrm{~L}$, it is not surprising that nearly all of our massively burned patients eventually require transfusion. We hypothesized that higher ratios of plasma transfusion during surgery would reduce coagulopathy and thereby decrease the requirement for RBC and plasma transfusion postoperatively. Because of the design of our study, we were not able to evaluate whether increased ratios of plasma conferred any mortality benefit. Though 148 operative interventions were included in the study, only 89 actual patients were enrolled. Therefore, any attempt to determine a mortality benefit had great potential for error.

While our research points to the possibility that plasma to RBC transfusion ratios $>1: 2$ are beneficial to burn patients, more research is necessary to determine potential detriments or whether this higher ratio confers any mortality benefit. Our research is also limited by the fact that it is a retrospective study rather than a prospective study. There is also the possibility that since the data for this study came from only one hospital, institutional practices might have influenced the results in a way that might make it difficult to reproduce the study results at another institution.

\section{Conclusions}

Higher intraoperative plasma to RBC ratios was associated with fewer transfusion requirements for plasma and RBCs in the first 24 hours postoperatively in our study. Patients who received a ratio $>1: 2$ of plasma to RBCs had significantly higher INRs preoperatively and longer surgeries, but still required significantly less plasma and RBCs postoperatively. These results may demonstrate the benefit of aggressive interoperative plasma use to correct perioperative coagulopathy. Further studies, preferably prospective studies, will be needed to know whether early, more aggressive plasma transfusion practices confer mortality benefits.

\section{References}

1. Miller RM (2004) Miller's Anesthesia. 6th edition Ch 47: Transfusion therapy 3376.

2. Tullis JL, Alving B, Bove JR (1985) Consensus conference. Fresh-frozen plasma. Indications and risks. JAMA 253: 551-553

3. Nuttall GA, Brost BC, Connis RT (2006) Practice guidelines for perioperative blood transfusion and adjuvant therapies: an updated report by the American Society of Anesthesiologists Task Force on Perioperative Blood Transfusion and Adjuvant Therapies. Anesthesiology. 105: 198-208.

4. Gonzalez EA, Moore FA, Holcomb JB, Miller CC, Kozar RA et al. (2007) Fresh Frozen Plasma Should be Given Earlier to Patients Requiring Massive Transfusion. J Trauma 62: 112-119.

5. Biffl WL, Smith WR, Moore EE, Gonzalez RJ, Morgan SJ, et al. (2001) Evolution of a multidisciplinary clinical pathway for the management of unstable patients with pelvic fractures. Ann Surg 233: 843-850.

6. Borgman MA, Spinella PC, Perkins JG, Grathwohl KW, Repine T, et al. (2007) The Ratio of Blood Products Transfused Affects Mortality in Patients Receiving Massive Transfusikons at a Combat Support Hospital. J Trauma 63: 805-813.

7. Hess JR, Holcomb JB (2008) Transfusion Practice in Military Trauma. Transfus Med 18: 143-150. 
Citation: Maani CV, Scott E, Desocio PA, McGhee LL, Garza T, et al. (2011) Intraoperative Plasma: Rbc Ratio Affects Postoperative Coagulopathy And Resuscitation Requirements. J Blood Disord Transfus 2:e101. doi:10.4172/2155-9864.1000e101

Page 3 of 3

8. Spinella PC, Perkins JG, Grathwohl KW, Beekley AC, Niles SE, et al. (2008) Effect of Plasma and Red Blood Cell Transfusions on Survival in Patients with Combat Related Traumatic Injuries. J Trauma 64: 69-77.

9. Holcomb JB, Wade CE, Michalek JE, Chisholm GB, Zarzabal LA, et al. (2008) Increased Plasma and Platelet to Red Blood Cell Ratios Improves Outcome in 466 Massively Transfused Civilian Trauma Patients. Ann Surg 248: 447-458.

10. Duchesne JC, Hunt JP, Wahl G, Marr AB, Wang YZ, et al. (2008) Review of
Current Blood Transfusion Strategies in a Mature Level 1 Trauma Center: Were We Wrong for the Last 60 Years? J Trauma 65: 272-276.

11. Kashuk JL, Moore EE, Johnson JL, Haenel J, Wilson M, et al. (2008) Postinjury Life Threatening Coagulopathy: Is 1:1 Fresh Frozen Plasma: Packed Red Blood Cells the Answer? J Trauma 65: 261-271.

12. Blumetti J, Hunt JL, Arnoldo BD, Parks JK, Purdue GF (2008) The Parkland Formula Under Fire: Is the Criticism Justified? J Burn Care Res 29: 180-186. 